\title{
Evaluation of Routine Health Examination for Screening Primary Open Angle Glaucoma in Eastern China: A Hospital-Based Cross-Sectional Study
}

This article was published in the following Dove Press journal:

Risk Management and Healthcare Policy

\author{
Yuan Fang ${ }^{l, *}$ \\ Jingyi Cheng $\mathbb{D}^{1, *}$ \\ Li Zhu' \\ Ming Xiao ${ }^{2}$ \\ Xinghuai Sun $\mathbb{D}^{1,3-5}$ \\ 'Department of Ophthalmology and \\ Visual Science, Eye and ENT Hospital, \\ Shanghai Medical College, Fudan \\ University, Shanghai 20003I, People's \\ Republic of China; ${ }^{2}$ Department of \\ Ophthalmology, Shanghai Bei Zhan \\ Hospital, Shanghai 200070, People's \\ Republic of China; ${ }^{3}$ Shanghai Key \\ Laboratory of Visual Impairment and \\ Restoration, Shanghai 20003I, People's \\ Republic of China; ${ }^{4} \mathrm{NHC}$ Key Laboratory \\ of Myopia, Chinese Academy of Medical \\ Sciences (Fudan University), Shanghai \\ 20003I, People's Republic of China; \\ ${ }^{5}$ State Key Laboratory of Medical \\ Neurobiology, Institutes of Brain Science \\ and Collaborative Innovation Center for \\ Brain Science, Fudan University, Shanghai \\ 200032, People's Republic of China
}

*These authors contributed equally to this work

\begin{abstract}
Purpose: To evaluate the effect of routine health examination (RHE) for screening primary open angle glaucoma (POAG) in Eastern China.
\end{abstract}

Materials and Methods: We enrolled patients with newly diagnosed and untreated POAG admitted to the Eye, Ear, Nose and Throat Hospital of Fudan University, Shanghai, China. Patients who were screened on RHE and then diagnosed with POAG were classified into the RHE group. The rest were classified as the non-RHE group. Demographic and ocular characteristics, 24-hour intraocular pressure (IOP), and glaucoma grade classified as the mild, moderate and severe groups according to glaucomatous optic neuropathy evaluated based on the enhanced Glaucoma Staging System were compared between the two groups. Results: In total, 172 patients with POAG (74 cases in the RHE group and 98 cases in the non-RHE group) were enrolled. The average age of patients in the RHE and non-RHE groups was $47.53 \pm 1.48$ years and $51.47 \pm 1.46$ years, respectively $(\mathrm{P}=0.064)$, while the average mean deviation (MD) was $5.98 \pm 0.66 \mathrm{~dB}$ and $9.79 \pm 0.80 \mathrm{~dB}(\mathrm{P}=0.001)$, respectively. The frequency of mild, moderate and severe grade glaucoma in the RHE group was $47.3 \%, 36.5 \%$, and $16.2 \%$, respectively, while that in the non-RHE group was $27.6 \%, 31.6 \%$, and $40.8 \%$, respectively $(\mathrm{P}=0.001)$. The presumed visual field MD progression rate for Chinese patients with untreated POAG was $0.97 \mathrm{~dB} /$ year. Additionally, the 24hour IOP curve in the two groups was similar, with the exception of the IOP at 10:00 PM and 2:00 AM, which was higher in the RHE group than that in the non-RHE group.

Conclusion: Our study showed RHE was an important and practical method to screen for POAG in Eastern China. RHE also assisted with an earlier diagnosis of POAG and may assist in preventing visual field loss.

Keywords: primary open angle glaucoma, screening, routine health examination, visual field progression

\section{Introduction}

Primary open angle glaucoma (POAG), a leading cause of blindness, is characterized by visual field loss due to irreversible retinal ganglion cell death. ${ }^{1}$ In China, the overall prevalence of POAG in 2015 was $1.02 \%$, ranging from $0.74 \%$ in individuals aged 40 to $3.02 \%$ in individuals aged 80 years in males and ranging from $0.54 \%$ to $2.24 \%$ in females. ${ }^{2}$ In China, the number of people with POAG is expected to increase from 6.06 million in 2020 to 9.59 million in $2050 .^{2}$ As most early cases are
Correspondence: Xinghuai Sun

Department of Ophthalmology and Visua Science, Eye and ENT Hospital, Shanghai

Medical College, Fudan University,

Shanghai 20003I, People's Republic of

China

Tel +862164377I34

Emailxhsun@shmu.edu.cn 
painless and asymptomatic, POAG is often diagnosed late. In developed western countries, more than half of POAG cases are undetectable. ${ }^{3-6}$ In Korean, among those diagnosed with POAG, only $8.0 \%$ patients were aware that they had the disease. ${ }^{7}$ In China, several population-based surveys showed that only $6.1-12 \%$ of patients with POAG had been previously diagnosed while the majority of patients were newly diagnosed during their participation in studies. ${ }^{8-11}$ The low rate of POAG diagnosis is a matter of public health concern.

Typically, there are two main sources of presumptive POAG cases in China. The first source is routine health examination (RHE) screening: individuals undergo a routine health examination, including direct ophthalmoscopy through un-dilated pupils, conducted by experienced ophthalmologists (usually not glaucoma specialists) in health examination centers. Individuals with a suspected glaucomatous change on the optic nerve will be referred to a hospital. Suspected glaucomatous optic neuropathy is defined as a vertical cup-to-disc ratio $(\mathrm{VCDR}) \geq 0.6, \mathrm{VCDR}$ difference $\geq 0.2$ between the eyes, minimal neural rim width $<0.1$ times the disc diameter, or disc hemorrhage. The second source is outpatient opportunistic screening (OOS, also referred to as non-RHE screening in this study): people visit a clinic or hospital for decreased vision, eye swelling, eye pain, dry eyes, or other symptoms and undergo slit-lamp examination, intraocluar pressure (IOP) measurement and fundus examination. Individuals with a suspected glaucomatous optic neuropathy, suspected retinal nerve fiber layer defect, or an IOP $>21 \mathrm{mmHg}$ are considered as presumptive glaucoma patients. All presumptive glaucoma patients are referred to a glaucoma specialists to receive a series of ocular examinations to ascertain the finally diagnosis. Although RHEs are thought to be helpful in screening for POAG, their actual effect on screening for POAG in clinics has not been evaluated to date.

Therefore, we conducted a cross-sectional hospitalbased study to identify patients with newly diagnosed and untreated POAG. We analyzed the differences between the patients referred by RHE (RHE group) and OOS (non-RHE group) to evaluate the effect of RHE on screening for POAG in Eastern China.

\section{Materials and Methods Study Design}

We conducted a cross-sectional, observational, hospitalbased, parallel-group study from 2013 to 2017 in the Eye, Ear, Nose and Throat (ENT) Hospital of Fudan
University, Shanghai, China, which is the biggest eye center in Eastern China.

\section{Patients}

Patients aged $\geq 30$ years with newly diagnosed and untreated POAG who were referred to the Eye and ENT Hospital of Fudan University in Shanghai, China were enrolled. Study participants were asked whether they had presumptive POAG on RHE screening. The diagnosis of POAG was confirmed by a glaucoma specialist after a comprehensive examination, which involved a structured ocular and systemic history taking and evaluation of the best corrected visual acuity (BCVA, E charts at a distance of 5 meters), slit lamp examination, Goldmann applanation tonometry (GAT, Haag Streit AG, Bern, Switzerland), gonioscopy, fundoscopy (CR-DGi Non-mydriatic retinal camera, Canon, Japan), central corneal thickness (CCT), axial length (AL), and reliable visual field testing (Humphrey automated perimetry, Carl Zeiss Medithech, Inc., Dublin, CA 30-2 threshold program or OCTOPUS 101 automated perimetry).

Visual-field defects defined by Humphrey automated perimetry had to meet at least two of the following criteria: (1) a cluster of three or more non-edge points, all of them depressed on the pattern deviation plot at a $\mathrm{P}<5 \%$ level and one of them depressed with $\mathrm{P}<1 \%$ level, (2) Glaucoma Hemifield test values outside the normal limits, (3) pattern standard deviation (PSD) outside $95 \%$ of normal limits (fixation losses $\leq 20 \%$, false-positive and falsenegative responses $\leq 15 \%$ ). Visual-field defects tested by OCTOPUS automated perimetry needed to have one spot depressed by $10 \mathrm{~dB}$, two contiguous spots depressed by 5 $\mathrm{dB}$ each, or three contiguous spots depressed by $2 \mathrm{~dB}$ each (Reliability Factor $\leq 15 \%$ ). ${ }^{1,12,13}$

Exclusion criteria for ocular conditions were previous intraocular surgery, history or signs of ocular inflammation, corneal conditions, or other anatomical abnormalities preventing reliable tonometry, and the use of any topical medication except artificial tears. Exclusion criteria for systemic conditions were as follows: diseases that could affect the optic nerve such as intracranial neoplasms, multiple sclerosis, or history of intracranial surgery history.

The initial IOP was defined as that obtained using GAT at the patient's first visit to the clinic during office hours.

\section{4-Hour IOP Measurements}

In all patients with newly diagnosed POAG in this study, 24-hour IOP measurements were obtained. Study 
participants were hospitalized to obtain 24-hour IOP measurements using a non-contact tonometer (NIDEK, Japan) by residents. The IOPs of both eyes were measured every two hours from 8:00 AM to 6:00 AM the next day. The subjects were instructed to sit or stand freely in the room until they went to bed after IOP measurements at 10:00 PM. From 00:00 AM to 6:00 AM, the participants were woken up every two hours and the IOP was measured instantly in the sitting position. Three measurements were taken for each eye at each time point, and the average values were adjusted by the CCT and then used for analysis. $^{14}$

The peak and trough IOPs were noted as the highest and lowest values among the 12 IOP values observed over the 24-hours of follow-up. IOP variation was calculated based on the difference between the peak and trough IOPs. The mean IOP was calculated as the average of IOP values at all time points, the diurnal mean IOP was calculated as the average of IOP values from 8:00 AM to 8:00 PM, and the nocturnal mean IOP was calculated as the average of IOP values from 10:00 PM to 6:00 AM. The mean diurnalto-nocturnal IOP change was determined by subtracting the nocturnal mean IOP from the diurnal mean IOP.

\section{Glaucoma Grade}

Glaucomatous optic neuropathy was evaluated based on the enhanced Glaucoma Staging System 2 (GSS 2). ${ }^{15}$ Briefly, according to the mean deviation (MD) and PSD or loss variance (LV) determined by the visual field tests, glaucomatous optic neuropathy can be classified from stage 0 to 5 . We formed subgroups as follows: stages $0-1,2-3$, and $4-5$ as the mild, moderate, and severe groups, respectively.

\section{POAG Subtypes}

In the 24-hour IOP curve, patients with a peak IOP $\leq$ $21 \mathrm{mmHg}$ in both eyes throughout the 24-hour period were diagnosed as having normal tension glaucoma (NTG). Those with a peak IOP $>21 \mathrm{mmHg}$ in either eye were diagnosed as having hypertension glaucoma (HTG). ${ }^{12}$

\section{Predicted Progression Model}

Since visual field progression in POAG is usually linear, the progression rate can be calculated through MD progression divided by the observation time. ${ }^{16-19}$ We estimated the progression rate based on the difference in the average MD between the RHE and the non-RHE groups divided by the difference of average years between the groups. We then set up a linear model to predict the average visual field progression rate in Chinese patients with untreated POAG.

\section{Data and Statistical Analysis}

For all eligible participants, only the right eye was included in the analysis. All data were collected and statistically analyzed using IBM SPSS Statistics (Version 25.0). Categorical variables were described using frequencies and constituent ratios, and chi-squared tests were used to determine the statistical significance. Continuous variables were described as means and the standard error of the means (SEM) and were compared using Student's t-tests. A P-value $<0.05$ was defined as statistically significant.

\section{Results}

Overall, 172 participants with POAG were enrolled into the study: 74 (43\%) and 98 (57\%) subjects were included in the RHE and non-RHE groups. As shown in Table 1, the demographic characteristics of those in the RHE and non-RHE groups did not significantly differ based on sex $(\mathrm{P}=0.759)$, systemic disease $(\mathrm{P}=0.596-0.89)$, smoking $(\mathrm{P}=0.741)$, or a family history of glaucoma $(\mathrm{P}=0.596)$. However, the mean ( \pm SEM) age in the RHE and nonRHE group was $47.53 \pm 1.48$ years and $51.47 \pm 1.46$ years $(\mathrm{P}=0.064)$, respectively.

The ophthalmic characteristics between the two groups neither significantly differed in terms of the high myopia ratio $(\mathrm{P}=0.978), \mathrm{AL}(\mathrm{P}=0.834)$ and initial $\mathrm{IOP}(\mathrm{P}=$ 0.829), nor did the CCT significantly differ between the groups $(\mathrm{P}=0.324)$. The mean $( \pm \mathrm{SEM})$ vertical cup/disk ratios in RHE and non-RHE group were $0.73 \pm 0.02$ and $0.80 \pm 0.06$, respectively $(\mathrm{P}=0.286)$. The mean $( \pm \mathrm{SEM})$ MD in the RHE group was significantly lower than that in the non-RHE group $(5.98 \pm 0.66 \mathrm{~dB}$ vs $9.79 \pm 0.80 \mathrm{~dB}$, $\mathrm{P}=0.001)$. Overall, $54.1 \%$ and $64.3 \%$ of patients in the RHE and non-RHE groups were diagnosed with NTG $(\mathrm{P}=0.175)$.

The glaucoma grades in all participants significantly differed between the RHE and the non-RHE groups $(\mathrm{P}=0.001)$. Overall, $47.3 \%, 36.5 \%$, and $16.2 \%$ of those in the RHE group and $27.6 \%, 31.6 \%$, and $40.8 \%$ of those in the non-RHE group had mild, moderate and severe glaucoma, respectively (Figure 1).

To exclude the deviation of CCT when measuring IOP, IOPs were corrected using CCT and were used in 
Table I Demographic and Ophthalmic Characteristics of Patients in RHE Group and Non-RHE Group

\begin{tabular}{|c|c|c|c|}
\hline & RHE Group & Non-RHE Group & P- value \\
\hline Number & 74 & 98 & \\
\hline Sex & & & 0.759 \\
\hline Male & $47(63.5)$ & $60(6 I .2)$ & \\
\hline Female & $27(36.5)$ & $38(38.8)$ & \\
\hline Age (years) & $47.53 \pm 1.48$ & $51.47 \pm 1.46$ & 0.064 \\
\hline \multicolumn{4}{|l|}{ Systemic diseases } \\
\hline Hypertension & $16(21.6)$ & $18(18.4)$ & 0.596 \\
\hline Diabetics & $7(9.5)$ & $8(8.2)$ & 0.765 \\
\hline Thyroid diseases & $2(2.7)$ & $3(3.1)$ & 0.89 \\
\hline Migraine & I (I.4) & $2(2.0)$ & 0.732 \\
\hline Smoking & $10(13.5)$ & $15(15.3)$ & $0.74 I$ \\
\hline Family history & $16(21.6)$ & I8 (18.4) & 0.596 \\
\hline High myopia & $22(29.7)$ & 31 (3I.6) & 0.978 \\
\hline Axial Length (mm) & $25.16 \pm 0.22$ & $25.22 \pm 0.19$ & 0.834 \\
\hline $\mathrm{CCT}(\mu \mathrm{m})$ & $540.93 \pm 4.15$ & $535.49 \pm 3.61$ & 0.324 \\
\hline Initial IOP (mmHg) & $\mid 6.69 \pm 0.41$ & $16.81 \pm 0.34$ & 0.829 \\
\hline Vertical C/D & $0.73 \pm 0.02$ & $0.80 \pm 0.06$ & 0.286 \\
\hline$M D(d B)$ & $5.98 \pm 0.66$ & $9.79 \pm 0.80$ & 0.001 \\
\hline Subtype & & & 0.175 \\
\hline HTG & $34(45.9)$ & $35(35.7)$ & \\
\hline NTG & $40(54.1)$ & $63(64.3)$ & \\
\hline
\end{tabular}

Notes: Statistical comparisons analyzed by chi-square test or Student's $t$-test. Data are described as number and proportions or means \pm standard error of the means. Abbreviations: RHE, routine health examination; CCT, central corneal thickness; IOP, intraocular pressure; C/D, cup to disk ratio; MD, mean deviation; HTG, hypertension glaucoma; NTG, normal tension glaucoma.

the 24-hour IOP analysis. ${ }^{11}$ The corrected IOPs in the two groups at 12 time points over 24-hours are compared in Figure 2. The shape of the IOP fluctuation over 24 hours was similar in the two groups: the IOP gradually decreased from the morning to the afternoon in the day time while the IOP increased slowly at night. The IOPs at night in the RHE group were lower than those in the non-RHE group. The mean ( \pm SEM) IOPs at 10:00 PM and 2:00 AM in RHE group were 13.22 \pm $0.45 \mathrm{mmHg}$ and $14.84 \pm 0.51 \mathrm{mmHg}$, respectively, while the mean ( \pm SEM) IOPs at 10:00 PM and 2:00 AM in the non-RHE group were $14.24 \pm 0.39 \mathrm{mmHg}$ and 16.11 $\pm 0.47 \mathrm{mmHg}$, respectively $(\mathrm{P}=0.09$ at $10: 00 \mathrm{PM}$ and $\mathrm{P}$ $=0.069$ at 2:00 AM, respectively). However, there were no significant differences in the IOPs between the two groups at any of the 12 time points.

Among those with the NTG subtype, the IOPs at night in the RHE group were lower than those in the non-RHE group (Figure 3 ). In particular, the mean ( \pm SEM) IOP at 10:00 PM in the RHE group was significantly lower than that in the non-RHE group $(11.5 \pm 0.49 \mathrm{mmHg}$ vs $13.28 \pm$ $0.039 \mathrm{mmHg}, \mathrm{P}=0.005)$.

Among those with the HTG subtype, the IOP at night in the RHE group was still lower than that in the non-RHE group (Figure 4). In particular, the mean ( \pm SEM) IOP at 2:00 AM in the RHE group was significantly lower than that in the non-RHE group $(16.75 \pm 0.69 \mathrm{mmHg}$ vs 19.16 $\pm 0.74 \mathrm{mmHg}, \mathrm{P}=0.044)$.

As shown in Table 2, the mean IOPs, the diurnal mean IOPs, and the nocturnal mean IOPs in the RHE group and the non-RHE group did not significantly differ. Additionally, there was no significant difference in the mean diurnal-to-nocturnal IOP change, peak IOP, trough IOP, and the IOP variation between the two groups. However, among those with the NTG subtype, the mean ( \pm SEM) IOP change in the RHE group was significantly differed from that in the non-RHE group $(0.20 \pm 0.28 \mathrm{mmHg}$ vs $-0.57 \pm 0.21 \mathrm{mmHg}, \mathrm{P}=0.029)$.

The average visual field progression rate in the untreated Chinese POAG patients was estimated as $0.97 \mathrm{~dB} /$ year (Figure 5). If these patients did not receive any treatment, the 


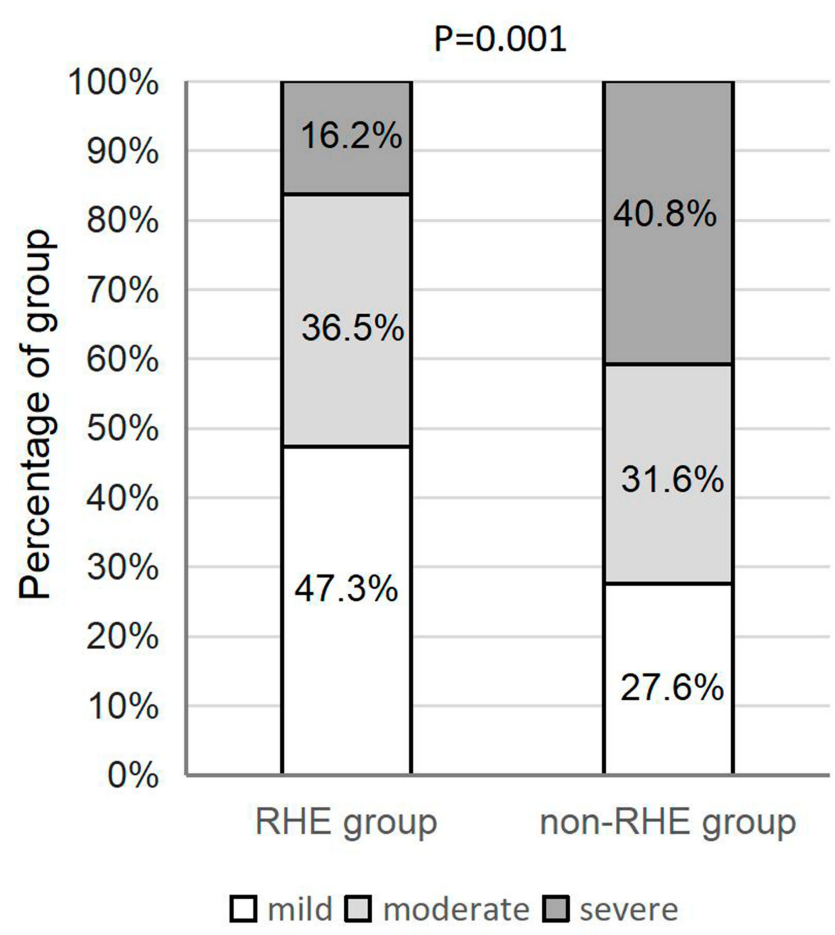

Figure I Proportion of mild, moderate, and severe glaucoma patients in the RHE and non-RHE groups. There were significant differences between the two groups $(P=0.001)$. Abbreviation: RHE, routine health examination.

predicted average age of patients with $\mathrm{MD}=20 \mathrm{~dB}$ was 62 years and the predicted average age of patients without a central visual filed was 72 years.

\section{Discussion}

POAG is a chronic irreversible optic nerve degeneration disease. ${ }^{1}$ Although the importance on the early diagnosis of POAG is well-known, the percentage of POAG patients with mild glaucomatous damage at the time of diagnosis is low globally. The most effective and cost-effective means of screening for POAG remains controversial. ${ }^{3}$ In China, RHE including direct ophthalmoscopy is an important method to screen for POAG. In our study, $43 \%$ of patients with newly diagnosed POAG were referred by RHE. However, the impact of RHE on screening POAG in China is still unknown. In this cross-sectional study, all patients with newly diagnosed and untreated POAG were divided into RHE and non-RHE groups. The data between these two groups were compared to evaluate the efficacy of RHE on the early diagnosis of POAG in Eastern China.

RHE can improve the rate of early POAG diagnosis. In our study, the average age of the RHE group was 3.94 years younger than that of the non-RHE group. Additionally, the MD of those in the RHE group was much lower than that of those in the non-RHE group (5.98 dB vs $9.79 \mathrm{~dB}$ ), showing that patients in the RHE group had less visual field loss. On comparing the glaucoma grade in these two groups, we found that $47.3 \%$ and $27.3 \%$ of those in the RHE and non-RHE groups had mild glaucoma. Additionally, $16.4 \%$ and $40.8 \%$ of those in the

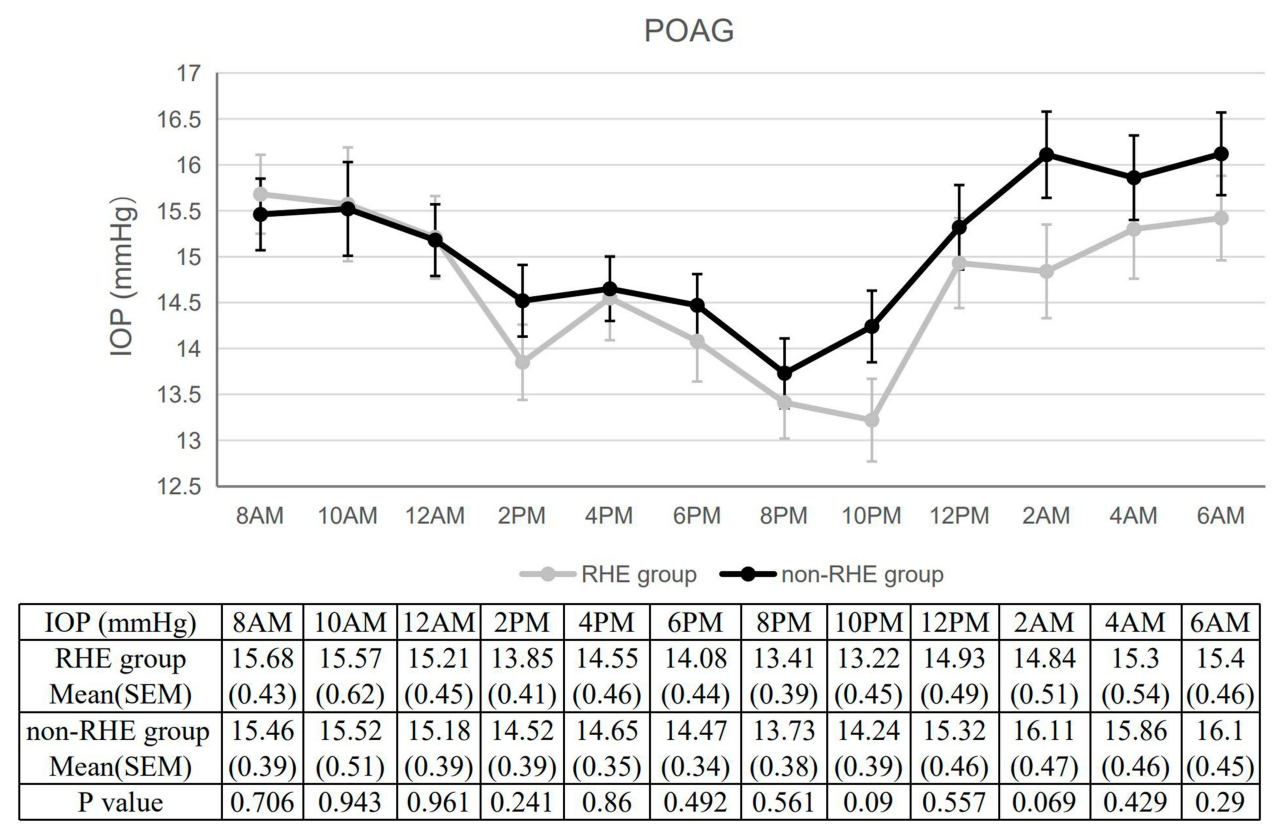

Figure 2 The 24-hour IOPs of patients with POAG in the RHE and non-RHE groups. At night, the IOPs in the RHE group were lower than those in the non-RHE group. However, the differences in IOP at all 12 time points between the two groups did not statistically differ.

Abbreviations: IOP, intraocular pressure; POAG, primary open angle glaucoma; RHE, routine health examination; SEM, standard error of the means. 


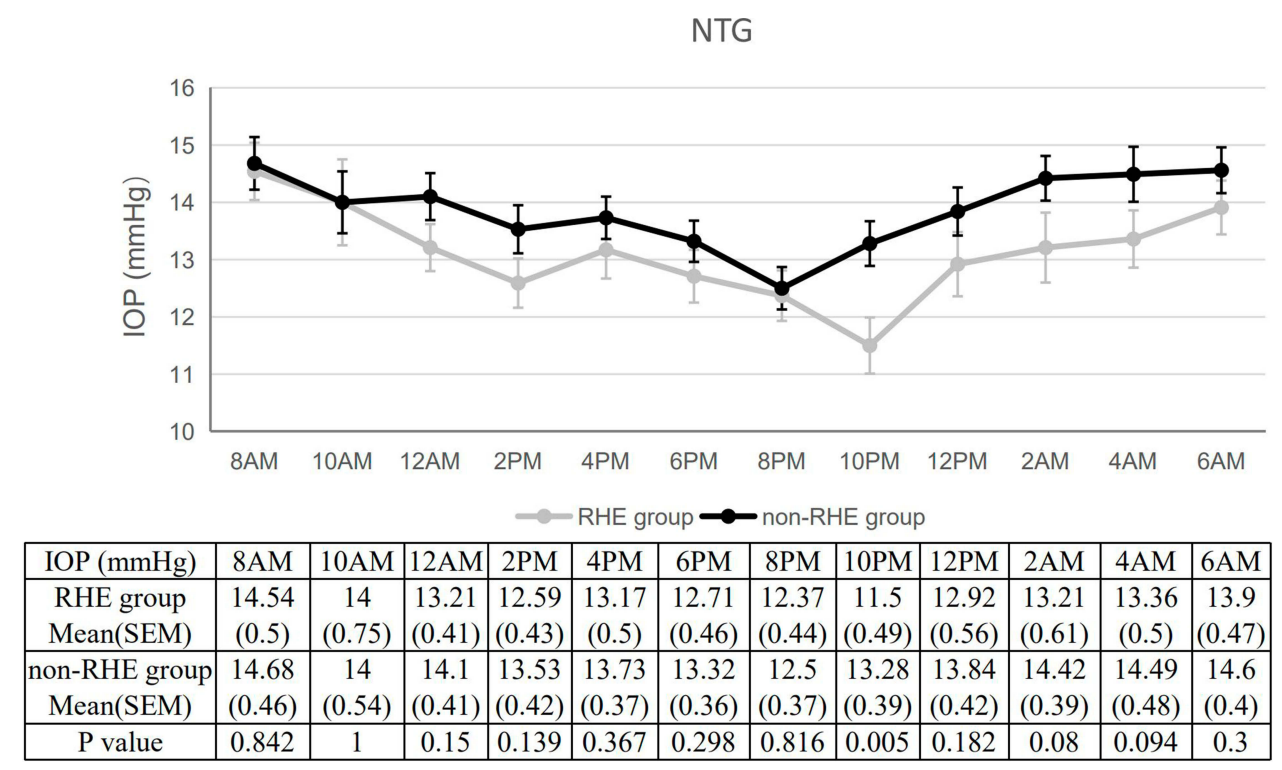

Figure 3 The 24-hour IOPs of patients with NTG in the RHE and non-RHE groups. During the nighttime, the IOPs in the RHE group were lower than those in the non-RHE group. The IOP at 10:00 PM significantly differed between the two groups $(P=0.005)$.

Abbreviations: IOP, intraocular pressure; NTG, normal tension glaucoma; RHE, routine health examination; SEM, standard error of the means.

RHE and non-RHE group had severe glaucoma. Our findings thus support the notion that RHE can significantly increase the rate of early POAG diagnosis. Our results are consistent with those from a Korean study in which the MD of newly diagnosed POAG patients referred by mass screening through the use of non-mydriatic fundus photography was much lower than those referred by opportunistic use of findings at primary eye clinic $(3.08 \mathrm{~dB}$ vs $6.70 \mathrm{~dB}) .^{7}$ Their results suggest that RHE screening, including non-mydriatic fundus examination, can increase the early POAG diagnosis when compare to non-RHE screening.

Early diagnosis is the prerequisite for early treatment. Several studies have showed that treatment of POAG can decrease the rate of visual field deterioration and extend patients' time in life with usable vision. ${ }^{20-24}$ Therefore, RHE screening for POAG is presumed to improve patients' quality of life.

HTG

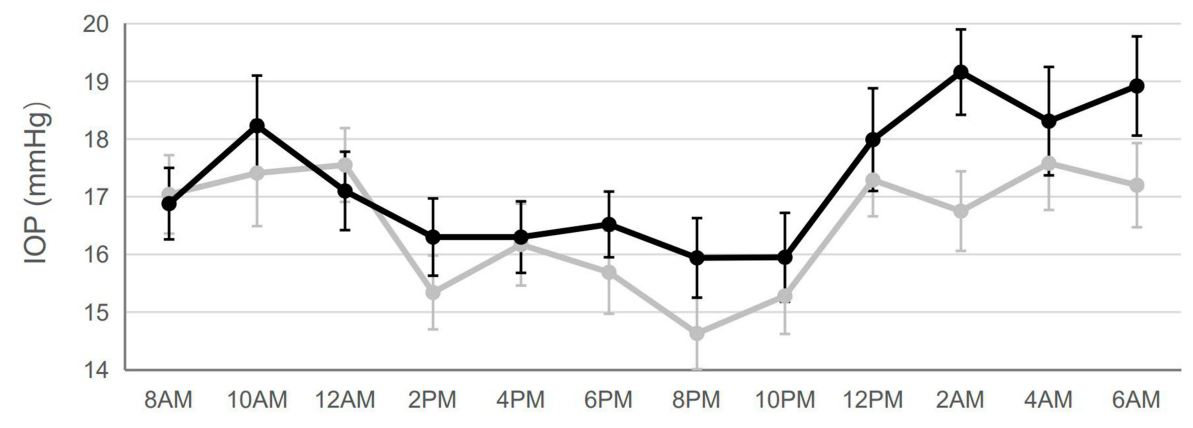

$\longrightarrow$ RHE group $\longrightarrow$ non-RHE group

\begin{tabular}{|c|c|c|c|c|c|c|c|c|c|c|c|c|}
\hline IOP $(\mathrm{mmHg})$ & 8AM & 10AM & 12AM & 2PM & 4PM & 6PM & 8PM & 10PM & 12PM & 2AM & 4AM & 6AM \\
\hline RHE group & 17.04 & 17.41 & 17.55 & 15.34 & 16.17 & 15.69 & 14.63 & 15.28 & 17.29 & 16.75 & 17.58 & 17.2 \\
Mean(SEM) & $(0.68)$ & $(0.92)$ & $(0.64)$ & $(0.64)$ & $(0.71)$ & $(0.72)$ & $(0.62)$ & $(0.66)$ & $(0.63)$ & $(0.69)$ & $(0.81)$ & $(0.73)$ \\
\hline non-RHE group & 16.88 & 18.23 & 17.1 & 16.3 & 16.3 & 16.52 & 15.94 & 15.95 & 17.99 & 19.16 & 18.31 & 18.9 \\
Mean(SEM) & $(0.62)$ & $(0.87)$ & $(0.68)$ & $(0.67)$ & $(0.62)$ & $(0.57)$ & $(0.69)$ & $(0.77)$ & $(0.89)$ & $(0.74)$ & $(0.94)$ & $(0.86)$ \\
\hline P value & 0.866 & 0.52 & 0.634 & 0.307 & 0.889 & 0.367 & 0.164 & 0.483 & 0.525 & 0.044 & 0.559 & 0.13 \\
\hline
\end{tabular}

Figure 4 The 24-hour IOPs of patients with HTG in the RHE and non-RHE groups. The IOP at 2:00 AM significantly differed between the two groups (P=0.044). Abbreviations: IOP, intraocular pressure; HTG, high tension glaucoma; RHE, routine health examination; SEM, standard error of the means. 


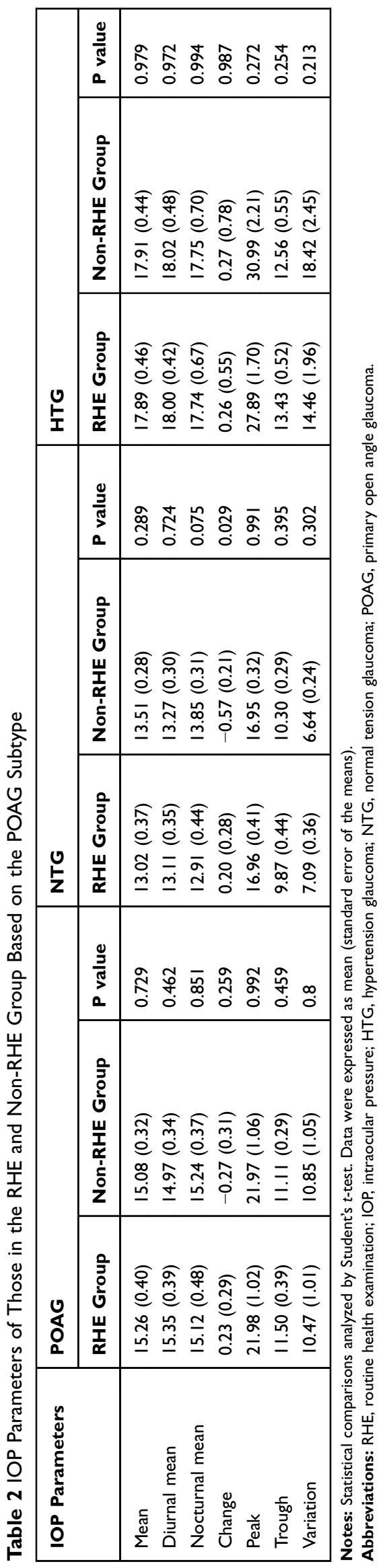

Both RHE and non-RHE screening depends on the primary evaluators. RHE screening usually only entails direct ophthalmoscopy through un-dilated pupils without the use of any other ocular instrument in health examination centers in China. It totally depends on the primary evaluators' observations. Conversely, there are some ocular instruments including slit-lamps, direct or indirect ophthalmoscopy, IOP measurement instruments, and the fundus camera which can be used in non-RHE screening. Primary evaluators in non-RHE screening can evaluate presumptive glaucoma patients using more reliable evidences. Therefore, RHE screening might produce a higher false-positive rate than non-RHE screening, as reported in a previous study. ${ }^{7}$

Twenty-four hour IOP measurements can provide more information than office-time IOP. In all patients with newlydiagnosed untreated POAG in our study, 159 (92.4\%) patients had an initial IOP $\leq 21 \mathrm{mmHg}$; only $13(7.6 \%)$ patients had an initial IOP $>21 \mathrm{mmHg}$. In the 24-hour IOP measurements, $103(59.9 \%)$ patients had a peak IOP $\leq 21 \mathrm{mmHg}$ and 69 (40.1\%) patients had a peak IOP $>21 \mathrm{mmHg}$. Approximately one third of patients with an initial IOP $\leq 21 \mathrm{mmHg}$ had a peak IOP $>21 \mathrm{mmHg}$ during the 24-hour IOP monitoring period. These findings showed that most peak IOPs occurred outside of office hours. Thus 24-hour IOP measurements can provide more information than those measured during office hours and can be used to devise more targeted treatment plan according to 24-hour IOP measures.

The trends in IOP among untreated POAG patients with disease progression are important to understand; however, there are limited data on these factors. The investigation of untreated unilateral POAG might provide us with some useful information. In previous studies, there were no significant differences in the 24-hour IOP monitoring parameters including the mean IOP, peak IOP, trough IOP, and the fluctuation in IOP (peak IOP- trough IOP) between the glaucomatous and non-glaucomatous eyes of unilateral POAG patients. ${ }^{25,26}$ Meanwhile, the vertical cup/disc ratios and the MD in glaucomatous and non-glaucomatous eye were $0.6 \pm 0.2$ versus 0.5 \pm 0.1 , and $5.41 \pm 4.67 \mathrm{~dB}$ versus $1.05 \pm 1.11 \mathrm{~dB}$, respectively. ${ }^{25,26}$ These studies suggested that there was no obvious change in the 24-hour IOP curve during the early stage of glaucoma. However, the 24-IOP trend during the middle stage of glaucoma remains unknown. In our cross-sectional study, we used the data from the RHE (early diagnosis group, average $\mathrm{MD}=5.98 \mathrm{~dB}$, vertical cup/disc ratio $=0.73$ ) and the non-RHE (late diagnosis group, average $\mathrm{MD}=9.79 \mathrm{~dB}$, vertical cup/disc ratio $=0.80$ ) groups to evaluate the IOP trends for untreated patients with POAG during the middle stage of glaucoma. The 


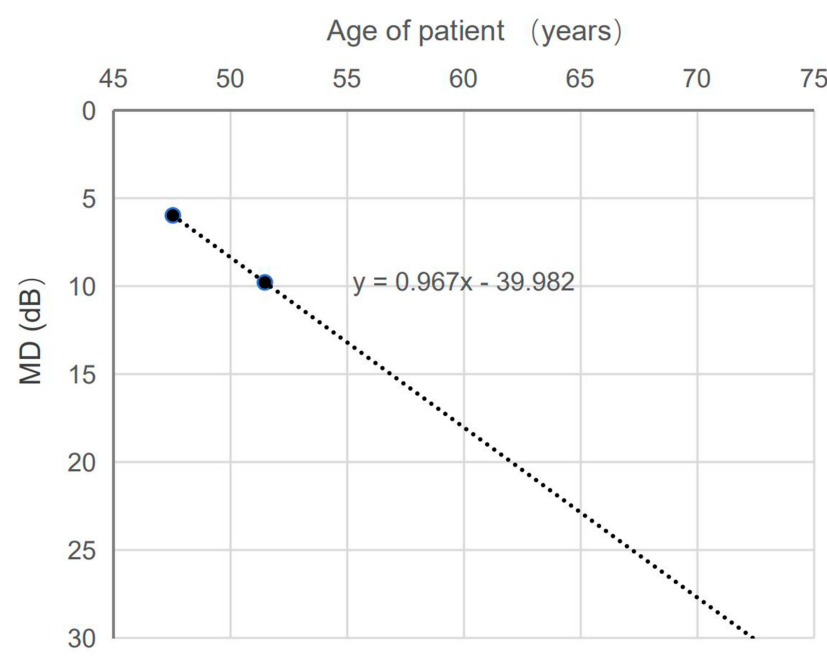

Figure 5 Predicted model of visual field defect progression with age. This model showed that the average progression rate was $0.967 \mathrm{~dB} /$ year and the predicted average age of patients without a central visual field was 72 years.

initial IOPs and the mean IOPs at 24 hours in the RHE and nonRHE groups were similar and consistent with the findings from previous studies. ${ }^{12,13,25-27}$ These findings suggested that the average IOP in the untreated POAG population did not significantly change as the disease progressed during the middle stage of glaucoma. However, the IOP at 10:00 PM and 2:00 $\mathrm{AM}$ in the non-RHE group was higher than that in the RHE group. Among the NTG patients, the IOP at 10:00 PM was significantly higher in the non-RHE group than in the RHE group. Additionally, in the HTG patients, the IOP at 2:00 AM was significantly higher in the non-RHE group than in the RHE group. These findings might suggest that IOP at 10:00 PM and 2:00 AM in untreated POAG patients might increase with diseases progression during the middle stage of glaucoma.

It is important to determine the likelihood of disease progression in order to properly manage patients with POAG. Many studies have found that visual field progression in POAG is usually linear and MD is an important index for visual field progression. ${ }^{16-19}$ A 12-year longitudinal study reported on untreated open angle glaucoma eyes with an average progressive MD deterioration of $0.96 \mathrm{~dB} /$ year. ${ }^{28}$ In the CNTGS study, the mean MD progression rate for untreated NTG was $0.41 \mathrm{~dB} /$ year. $^{2,9}$ Additionally, in the EMGT study, the mean MD progression rates for untreated open angle glaucoma were $1.08 \mathrm{~dB} /$ year overall, and 1.31 $\mathrm{dB} /$ year, $0.36 \mathrm{~dB} /$ year, and $3.13 \mathrm{~dB} /$ year for HTG, NTG, and pseudoexfoliation glaucoma, respectively. ${ }^{30}$ Progression was considerably and significantly faster in older patients. ${ }^{2,9,30}$ In this study, we found that the progression rate for untreated POAG patients was $0.97 \mathrm{~dB} /$ year, which was similar to that previously reported. ${ }^{28,30}$ These findings suggest that untreated POAG patients in China have similar progression patterns to patients in western countries, which might assist Chinese doctors in evaluating the efficacy of POAG treatment in the future. For patients without specific data on visual field progression during their untreated periods, their visual field progression rates can be compared with $0.97 \mathrm{~dB} /$ year to evaluate the effect of treatment. Moreover, the optimal duration between two visual field examinations for presumptive POAG cases with an initial IOP $\leq 21 \mathrm{mmHg}$ might be suggested to be 1 year. An interval of 3-6 months might be too small to detect visual field progression.

This study was subject to several limitations which merit mentioning here. First, it did not investigate the total number of presumptive POAG patients referred by RHE or non-RHE. Additionally, the accuracy and specificity of the two screening methods cannot be compared in this study. Second, we did not compare the cost-effectiveness of the two screening methods, this might be an important factor to consider in public health programme planning. Third, the visual progression rate was evaluated by the difference of the age and MD in the RHE and nonRHE groups rather than the comparison of progression within the same patients. This value was therefore indirectly estimated rather than directly calculated. The true progression rate in untreated Chinese POAG patients still requires further investigation.

In conclusion, this study showed that RHE was an important and practical method to screen for POAG in Eastern China. RHE can assist in an earlier diagnosis of POAG and might prevent more visual field loss. Moreover, the IOP at 2:00 AM or 10:00 PM in the untreated POAG eyes was estimated to increase with disease progression in the middle stage of glaucoma. Additionally, the visual field progression rate among this cohort of Chinese patients with POAG was similar to that reported in western countries. However, only $43 \%$ of the newly diagnosed patients with POAG in this study were referred by RHE to our hospital in Shanghai, which is one of the most developed areas in China. We presume that the proportion of newly diagnosed patients with POAG referred by RHE in China is lower than that reported in this study. Thus, efforts are needed to educate people regarding the effectiveness of RHE on POAG screening. Broader use of RHE will benefit the earlier diagnosis of other ocular diseases, leading to a potential future reduction in disease burden. Additionally, it could sequentially improve patients' quality of life. 


\section{Data Sharing Statement}

The datasets used and/or analyzed during the current study are available from the corresponding author on reasonable request.

\section{Ethics Approval and Consent}

This study was approved by the Ethics Committee of Eye \& ENT Hospital and followed the tenets of the Declaration of Helsinki. Informed consent was obtained from all participants. Consent for publication was obtained from all participants.

\section{Author Contributions}

All authors made substantial contributions to conception and design, acquisition of data, or analysis and interpretation of data; took part in drafting the article or revising it critically for important intellectual content; gave final approval of the version to be published; and agree to be accountable for all aspects of the work.

\section{Funding}

This work was supported by the International Science \& Technology Cooperation Program of China (No.2015DFA31340), National Natural Science Foundation (NSFC81100667), the State Key Program of National Natural Science Foundation of China (81430007), the top priority of clinical medicine center of Shanghai (2017ZZ01020).

\section{Disclosure}

The authors report no conflicts of interest in this work.

\section{References}

1. European Glaucoma Society. Terminology and Guidelines for Glaucoma. 4th ed. Savona, Italy: PubliComm; 2014.

2. Song P, Wang J, Bucan K, Theodoratou E, Rudan I, Chan KY. GHERG (2017) national and subnational prevalence and burden of glaucoma in China: a systemic analysis. J Glob Health. 2017;7 (2):020705. doi:10.7189/jogh.07.020705

3. Quigley HA. Number of people with glaucoma worldwide. $\mathrm{Br} J$ Ophthalmol. 1996;80(5):389-393. doi:10.1136/bjo.80.5.389

4. Quigley HA, West SK, Rodriguez J, Munoz B, Klein R, Snyder R. The prevalence of glaucoma in a population-based study of hispanic subjects: proyecto VER. Arch Ophthalmol. 2001;119(12):1819-1826. doi:10.1001/archopht.119.12.1819

5. Burr JM, Mowatt G, Hernández R, et al. The clinical effectiveness and cost-effectiveness of screening for open angle glaucoma: a systematic review and economic evaluation. Health Technol Assess. 2007;11(41): iii-iv, ix-x, 1-190. doi:10.3310/hta11410

6. Scuderi GL, Cedrone C, Nucci C, Macri G, Moretto C, Cerulli L. The prevalence of primary open angle glaucoma in Italy. Int J Ophthalmol. 2003;3(1):99.
7. Song YJ, Kim YW, Park KH, Kim YK, Choi HJ, Jeoung JW. Comparison of glaucoma patients referred by glaucoma screening versus referral from primary eye clinic. PLoS One. 2019;14(1): e0210582. doi:10.1371/journal.pone.0210582

8. Sun J, Zhou X, Kang Y, et al. Prevalence and risk factors for primary open-angle glaucoma in a rural northeast China population: a population-based survey in Bin County, Harbin. Eye. 2012;26(2):283-291. doi:10.1038/eye.2011.243

9. He J, Zou H, Lee RY, et al. Prevalence and risk factors of primary open-angle glaucoma in a city of Eastern China: a population-based study in Pudong New District, Shanghai. BMC Ophthalmol. 2015;15:134. doi:10.1186/s12886-015-0124-x

10. He M, Foster PJ, Ge J, et al. Prevalence and clinical characteristics of glaucoma in adult Chinese: a population-based study in Liwan District, Guangzhou. Invest Ophthalmol Vis Sci. 2006;47(7):27822788. doi:10.1167/iovs.06-0051

11. Xu L, Chen JH, Li JJ, et al. The prevalence and its screening methods of primary open angle glaucoma in defined population based study of rural and urban in Beijing. Chin J Ophthalmol. 2004;40:726-732.

12. Cheng J, Kong X, Xiao M, Sun X. Twenty-four-hour pattern of intraocular pressure in untreated patients with primary open-angle glaucoma. Acta Ophthalmol. 2016;94(6):e460-e467. doi:10.1111/aos

13. Quaranta L, Katsanos A, Riva I, et al. Twenty-four-hour intraocular pressure and ocular perfusion pressure characteristics in newly diagnosed patients with normal tension glaucoma. Eye (Lond). 2016;30 (11):1481-1489. doi:10.1038/eye.2016.168

14. Ehlers N, Bramsen T, Sperling S. Applanation tonometry and central corneal thickness. Acta Ophthalmol. 2009;53(1):34-43. doi:10.1111/ j.1755-3768.1975.tb01135.x

15. Brusini P, Filacorda S. Enhanced glaucoma staging system(GSS2) for classifying functional damage in glaucom. J Glaucoma. 2006;15 (1):40-46. doi:10.1097/01.ijg.0000195932.48288.97

16. Rasker MT, van den Enden A, Bakker D, Hoyng PF. Rate of visual field loss in progressive glaucoma. Arch Ophthalmol. 2000;118 (4):481-488. doi:10.1001/archopht.118.4.481

17. Pereira ML, Kim CS, Zimmerman MB, Alward WL, Hayreh SS, Kwon YH. Rate and pattern of visual field decline in primary openangle glaucoma. Ophthalmology. 2002;109(12):2232-2240. doi:10.1016/s0161-6420(02)01248-4

18. Bengtsson B, Patella VM, Heijl A. Prediction of glaucomatous visual field loss by extrapolation of linear trends. Arch Ophthalmol. 2009;127(12):1610-1615. doi:10.1001/archophthalmol.2009.297

19. Smith SD, Katz J, Quigley HA. Analysis of progressive change in automated visual fields in glaucoma. Invest Ophthalmol Vis Sci. 1996;37(7):1419-1428. doi:10.1016/S0002-9394(14)72089-6

20. Group CN-TGS. Comparison of glaucomatous progression between untreated patients with normal-tension glaucoma and patients with therapeutically reduced intraocular pressures. Am J Ophthalmol. 1998;126(4):487-497. doi:10.1016/s0002-9394(98)00223-2

21. Group CN-TGS. The effectiveness of intraocular pressure reduction in the treatment of normal-tension glaucoma. Am J Ophthalmol. 1998;126(4):498-505. doi:10.1016/s0002-9394(98)00272-4

22. Heijl A, Leske MC, Bengtsson B, Hyman L, Bengtsson B, Hussein M; Early Manifest Glaucoma Trial Group. Reduction of intraocular pressure and glaucoma progression: results from the early manifest glaucoma trial. Arch Ophthalmol. 2002;120(10):1268-1279. doi:10.1001/archopht.120.10.1268

23. Leske MC, Heijl A, Hussein M, Bengtsson B, Hyman L, Komaroff E; Early Manifest Glaucoma Trial Group. Factors for glaucoma progression and the effect of treatment: the early manifest glaucoma trial. Arch Ophthalmol. 2003;121(1):48-56. doi:10.1001/ archopht.121.1.48

24. Hyman LG, Komaroff E, Heijl A, Bengtsson B, Leske MC; Early Manifest Glaucoma Trial Group. Treatment and vision-related quality of life in the early manifest glaucoma trial. Ophthalmology. 2005;112 (9):1505-1513. doi:10.1016/j.ophtha.2005.03.028 
25. Lin Z, Huang S, Huang P, Li C, Chen Z, Zhong Y. Concordance of 24-h intraocular pressure curve in patients with untreated unilateral primary open-angle glaucoma. Exp Ther Med. 2018;16(2):14611469. doi:10.3892/etm.2018.6315

26. Wang NL, Friedman DS, Zhou Q, et al. A population-based assessment of 24-hour intraocular pressure among subjects with primary open-angle glaucoma: the Handan Eye Study. Invest Ophthalmol Vis Sci. 2011;52(11):7817-7821. doi:10.1167/iovs.11-7528

27. Deokule SP, Doshi A, Vizzeri G, et al. Relationship of the 24-hour pattern of intraocular pressure with optic disc appearance in primary open angle glaucoma. Ophthalmology. 2009;116(5):833-839. doi:10.1016/j.ophtha.2008.10.034
28. Katz J, Gilbert D, Quigley HA, Sommer A. Estimating progression of visual field loss in glaucoma. Ophthalmology. 1997;104(6):10171025. doi:10.1016/S0161-6420(97)30192-4

29. Anderson DR, Drance SM, Schulzer M; Collaborative NormalTension Glaucoma Study Group. Natural history of normal-tension glaucoma. Ophthalmology. 2001;108(2):247-253. doi:10.1016/ s0161-6420(00)00518-2

30. Heijl A, Bengtsson B, Hyman L, Leske MC; Early Manifest Glaucoma Trial Group. Natural history of open angle glaucoma. Ophthalmology. 2009;116(12):2271-2276. doi:10.1016/j.ophtha.2 009.06 .042
Risk Management and Healthcare Policy

\section{Publish your work in this journal}

Risk Management and Healthcare Policy is an international, peerreviewed, open access journal focusing on all aspects of public health, policy, and preventative measures to promote good health and improve morbidity and mortality in the population. The journal welcomes submitted papers covering original research, basic science, clinical \& epidemiological studies, reviews and evaluations,

\section{Dovepress}

guidelines, expert opinion and commentary, case reports and extended reports. The manuscript management system is completely online and includes a very quick and fair peer-review system, which is all easy to use. Visit http://www.dovepress.com/testimonials.php to read real quotes from published authors. 